\title{
A Prospective Randomized Clinical Trial Comparing Bone Union Rate Following Anterior Cervical Discectomy and Fusion Using a Polyetheretherketone Cage: Hydroxyapatite/ B-Tricalcium Phosphate Mixture versus Hydroxyapatite/Demineralized Bone Matrix Mixture
}

\author{
Jemin $\mathrm{Yi}^{1}$, Gun Woo Lee ${ }^{2}$, Woo Dong Nam ${ }^{1}$, Kye Young Han ${ }^{1}$, Myung-Ho Kim ${ }^{3}$, \\ Jong Won Kang ${ }^{4}$, Jonghwa Won ${ }^{5}$, Seong Wan Kim ${ }^{6}$, Won Noh ${ }^{7}$, Jin S Yeom ${ }^{8}$ \\ ${ }^{1}$ Department of Orthopaedic Surgery, Kangwon National University College of Medicine, Chuncheon, Korea \\ ${ }^{2}$ Department of Orthopaedic Surgery, Armed Forces Yangju Hospital, Yangju, Korea \\ ${ }^{3}$ Department of Orthopaedic Surgery, Dankook University College of Medicine, Cheonan, Korea \\ ${ }^{4}$ Department of Orthopaedic Surgery, Sun General Hospital, Daejeon, Korea \\ ${ }^{5}$ Department of Orthopaedic Surgery, Chamjoeun Hospital, Gwangju, Korea \\ ${ }^{6}$ Department of Orthopaedic Surgery, National Police Hospital, Seoul, Korea \\ ${ }^{7}$ Department of Orthopaedic Surgery, Uijung-bu Chuk Hospital, Uijung-bu, Korea \\ ${ }^{8}$ Spine Center and Department of Orthopaedic Surgery, Seoul National University Bundang Hospital, \\ Seoul National University College of Medicine, Sungnam, Korea
}

Study Design: Prospective randomized noninferiority trial.

Purpose: To evaluate whether the union rate of anterior cervical discectomy and fusion (ACDF) using a polyetheretherketone (PEEK) cage filled with a mixture of hydroxyapatite (HA) and demineralized bone matrix (DBM) is inferior to that of a mixture of $\beta$-tricalcium phosphate ( $\beta$-TCP) and HA.

Overview of Literature: There have been no clinical trials investigating the outcomes of a mixture of HA and DBM in a PEEK cage in ACDF.

Methods: Eighty-five eligible patients were randomly assigned to group $B(n=43)$, in which a PEEK cage with a mixture of HA and DBM was used, or group $C(n=42)$, in which a PEEK cage with a mixture of HA and $\beta$-TCP was used. The primary study endpoint was the fusion rate, which was assessed with dynamic radiographs and computed tomography (CT) scans. Secondary endpoints included pain intensity using a visual analogue scale, functional outcome using a neck disability index score, laboratory tests of inflammatory profiles, and the infection rate.

Results: Seventy-seven patients (38 in group B and 39 in group C) were included in the final analysis. One year postoperatively, bone fusion was achieved in $87 \%$ of group B patients and $87 \%$ of group $C$ patients on dynamic radiographs, and $87 \%$ of group B patients and $72 \%$ of group $C$ patients on CT scans ( $p=1.00$ and 0.16 , respectively). There were also no between-groups differences with

Received Dec 14, 2014; Revised Dec 15, 2014; Accepted Dec 24, 2014

Corresponding author: Gun Woo Lee

Department of Orthopaedic Surgery, Armed Forces Yangju Hospital,

461 Yongam-ri, Eunhyeon-myeon, Yangju 482-863, Korea

Tel: +82-31-857-1319, Fax: +82-31-857-6911, E-mail: gwlee1871@gmail.com 
respect to the secondary endpoints.

Conclusions: A HA/DBM mixture inside a PEEK cage can provide noninferior outcomes compared to a HA/TCP mixture in ACDF.

Keywords: Anterior cervical discectomy and fusion; Polyetheretherketone cage; Hydroxyapatites; Demineralized bone matrix; Betatricalcium phosphate

\section{Introduction}

Achieving a solid bony union is critical to obtaining an excellent outcome following anterior cervical discectomy and fusion (ACDF). Although the tricortical iliac autograft has been the gold standard graft material for fusion, graft harvest may lead to donor site morbidity such as pain, infection, fracture, loss of sensation, meralgia paresthetica, and hematomas [1-4]. Allografts also have several drawbacks, including fragility or graft fractures, contamination, and disease transmission [1,4-7]. In this regard, the use of polyetheretherketone (PEEK) cages may be a reasonable alternative to help avoid such problems. However, the PEEK cage does not have osteogenic ability per se $[5,8,9]$. Therefore, it needs to be filled with some material, such as a bone graft substitute, to enhance bone regeneration.

Biologically, ideal graft materials or graft substitutes should have three basic properties: osteogenicity, osteoconductivity, and osteoinductivity. A mixture of hydroxyapatite (HA) and $\beta$-tricalcium phosphate $(\beta$-TCP) has been widely used for bone regeneration due to its several positive physical properties $[1,4,7,10,11]$. Both HA and $\beta$-TCP share chemical similarities with human bone minerals and therefore show excellent compatibility; in addition, they also have superior osteoconductivity $[1,2,4,5,10-14]$. On the other hand, demineralized bone matrix (DBM) has also been used as a graft substitute because it leads to superior bony fusion, owing mainly to its osteoinductive ability $[1,4,12,15-19]$. Whereas HA and $\beta$-TCP have osteoconductive properties and DBM has osteoinductive properties, a single graft material that fully incorporates both properties has not yet been developed. The use of a mixture of HA and DBM as a filler within the PEEK cage may theoretically provide both osteoinductivity and osteoconductivity. However, to the best of our knowledge, there have been no clinical trials to evaluate the outcomes of this mixture in a PEEK cage. Therefore, in a prospective randomized trial, we analyzed the radiological and clinical outcomes of ACDF using a PEEK cage filled with a mixture of HA and DBM (HA/DBM, Bonion, CG Bio Inc., Seoul, Korea) and compared it to the outcomes from using a PEEK cage filled with a mixture of HA and $\beta$-TCP (HA/ $\beta$-TCP, Cervios ChronOs, Synthes, West Chester, PA, USA), which is widely recognized as an effective fusion material. We hypothesized that the HA/DBM mixture as a graft material within a PEEK cage would result in noninferior efficacy compared to the HA/ $\beta$-TCP mixture in terms of the union rates and clinical outcomes of ACDF.

\section{Materials and Methods}

\section{Participants}

This study was approved by the Institutional Review Board of the Seoul National University Bundang Hospital and was conducted following approval of the health authority by the Korea Food and Drug Administration. The trial has been registered in the database ClinicalTrials.gov (NCT01615328).

This study was conducted only on the patients who fully fit within the following inclusion and exclusion criteria. The inclusion criteria were: (1) patients with a herniated cervical intervertebral disc, cervical spondylotic radiculopathy and/or myelopathy, which were diagnosed using cervical spine radiographs and magnetic resonance images (MRI) that corresponded to clinical manifestations and physical examinations; (2) patients who underwent single-level ACDF; (3) patients aged between 20 years and 80 years; (4) patients who volunteered for this study and gave written informed consent; and (5) patients who were followed up for one year or more. Those who met any of the following criteria were excluded: (1) patients with fractures, infection, or tumors in the cervical spine; (2) patients with osteoporosis, which was diagnosed as a 
T-score $<-2.5$ in dual-energy X-ray absorptiometry bone densitometry taken on the hip; (3) patients with hemorrhagic disorders such as hemophilia and thrombocytopenia; (4) patients with a follow-up period of less than one year; and (5) patients who were not suitable for this study as judged by the principal investigator (the last author).

\section{Randomization}

Patients were randomly assigned into a study group (group B), for which a Bonion PEEK cage filled with a mixture of HA and DBM was used, or a control group (group C), for which a Cervios ChronOs PEEK cage filled with a mixture of HA and $\beta$-TCP was used. A randomization table was produced by a computer-generated allocation program (nQquery Advisor 7.0, Statistical Solutions, Cork, Ireland). Randomization was stratified by four variables: (1) age (over vs. under 60.0 years); (2) gender (male vs. female); (3) smoking status (smoker vs. non-smoker); and (4) operation level (C3/4/5/6 vs. C6/7/ T1). One of the research coordinators opened the randomization table just before the surgery, and the surgeon was informed of the random allocation sequence after the surgical approach was completed.

\section{Surgical procedures and postoperative management protocols}

All operations were performed by one surgeon (the last author) using the same surgical technique. A standard Smith-Robinson approach to the cervical spine was carried out through a transverse incision. After removal of the disc material, the endplates were prepared with a quadrangular curette and a pituitary rongeur, and the neural structures were decompressed sufficiently with or without uncoforaminotomy, as required. Great care was taken to remove the intradiscal cartilaginous tissues, but the endplates were not decorticated. A PEEK cage, the type of which was predetermined by the randomization procedure, was inserted gently while the interbody space was distracted. The choice of cage height depended on the intraoperative estimate made using cage trials. Rigid anterior plate-screw fixation (Atlantis, Medtronic, Memphis, TN, USA) was performed in all patients.

Patients in both groups were treated with the same postoperative protocols. All patients were allowed to ambulate on the first day after surgery, and the majority were discharged from the hospital on the fifth postoperative day. Patients were instructed to keep the Miami cervical brace in place for three months.

\section{Outcome measures}

The primary endpoint was the bone fusion rate at six and 12 months after surgery. A bony union was defined as a difference in the interspinous distances (ISDs) (Fig. 1) of $2 \mathrm{~mm}$ or less on dynamic lateral radiographs taken in flexion and extension, and the definite presence of a continuous fusion mass either inside or outside the cage on sagittal and coronal reconstructed computed tomography (CT) scans (Fig. 2). A difference in ISDs greater than 2 $\mathrm{mm}$, as well as an indefinite fusion mass on CT scans, was defined as a nonunion (Fig. 3).

All dynamic radiographs for determining fusion status were obtained at three, six and 12 months after surgery. All measurement with dynamic radiographs was performed by three orthopedic spine surgeons who were not involved in the treatment of the patients. The measurement was carried out using the PACS (Infinitt, Bracknell, Berkshire, UK). The average value of the differences in ISDs measured by the three surgeons was used to determine the bony union status.

CT scans were obtained at six and 12 months after surgery, and measurements were performed with three-

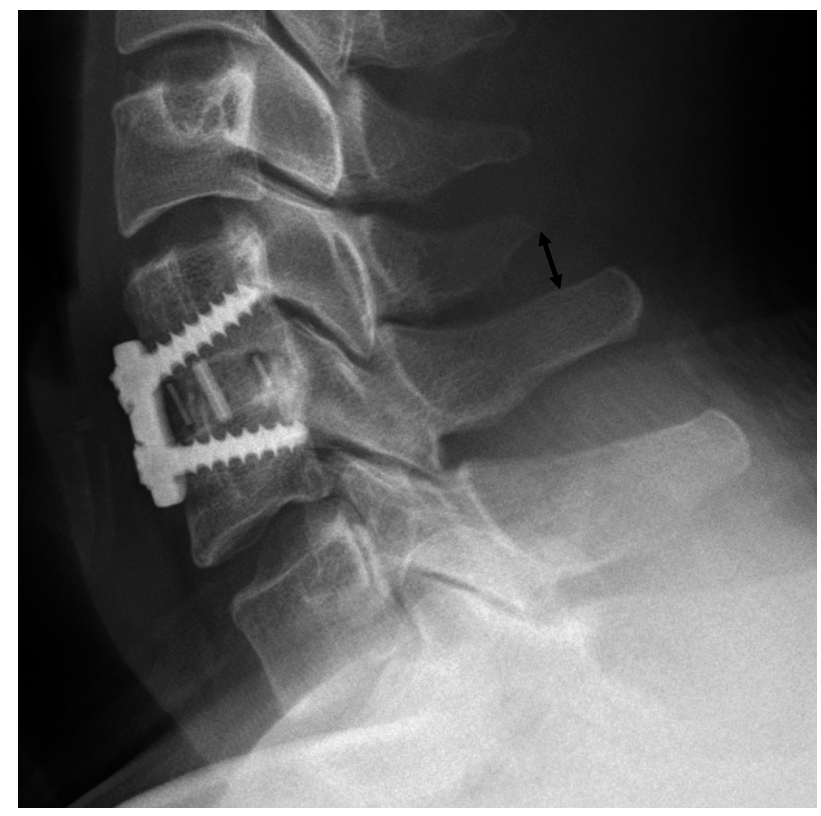

Fig. 1. The method of measuring interspinous distance (arrow) is shown. 
dimensional surgical simulation software (V-works, Cybermed Inc., Reston, VA, USA) to verify the fusion mass
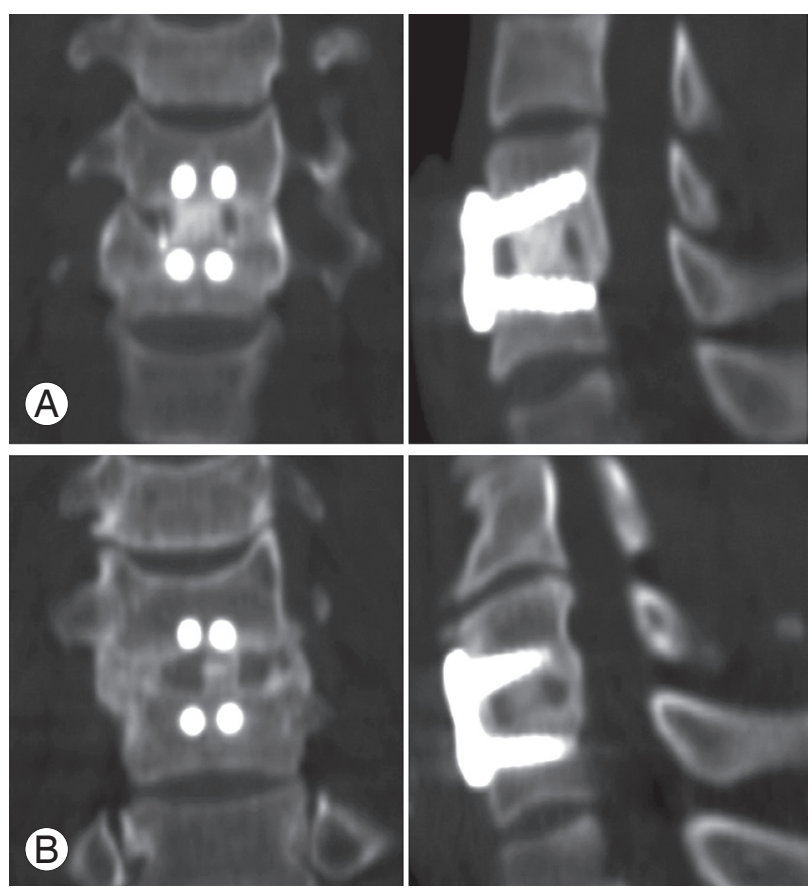

Fig. 2. Coronal and sagittal computed tomography scans taken 12 months after surgery showing satisfactory unions. (A) One case in group $B$ and $(B)$ one case in group $C$ are shown.
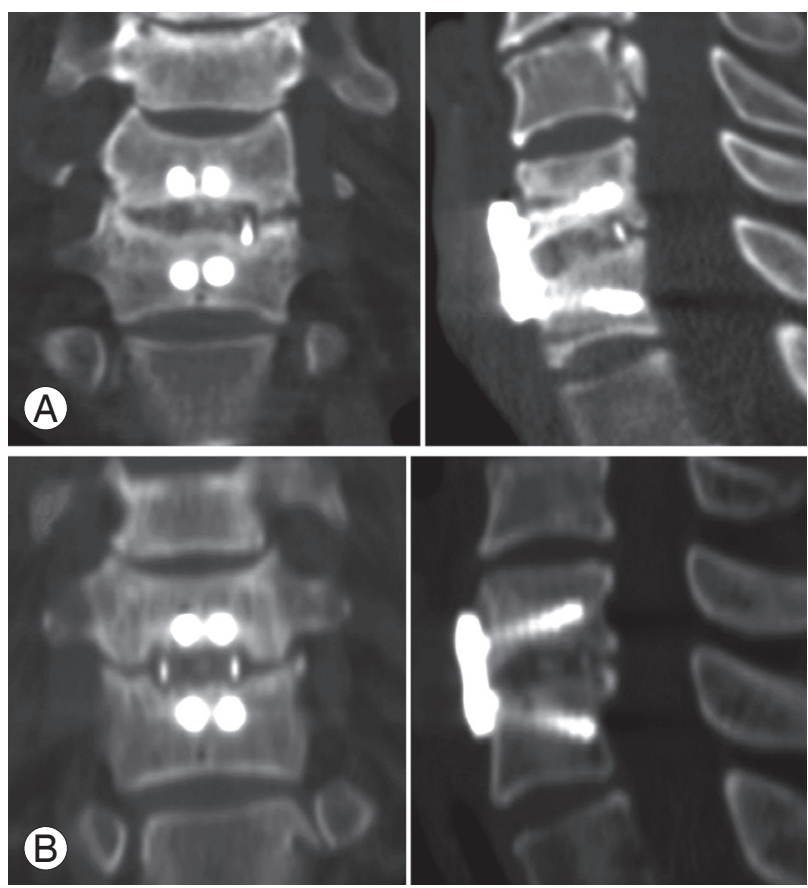

Fig. 3. Coronal and sagittal computed tomography scans taken 12 months after surgery showing nonunions. (A) One case in group B and (B) one case in group $\mathrm{C}$ are shown. in more detail, and the fusion status was judged by the agreement of three orthopedic surgeons who were not involved in the treatment of the patients.

The secondary endpoints were: (1) the intensity of posterior neck pain and pain radiating to the upper extremity based on a 10-point visual analogue scale (VAS); (2) the Neck Disability Index (NDI); (3) laboratory tests of inflammatory profiles, including erythrocyte sedimentation rate (ESR), C-reactive protein (CRP), and white blood cell count (WBC); and (4) the infection rate. These data were collected and tabulated prospectively by a research coordinator preoperatively and at each follow-up visit at one, three, six, and 12 months postoperatively.

\section{Sample size estimation}

This study was conducted to determine whether the HA/ DBM mixture as a fusion material inside a PEEK cage is inferior to the HA/ $\beta$-TCP mixture based on union rates and clinical outcomes, representing a noninferiority trial. Consequently, we used the noninferiority criterion to calculate the appropriate sample size [20]. The primary endpoint was the union rate, and the expected union rate of the control group (group C) was $93 \%$, which is in agreement with the study of Chou et al. [21]. To determine the noninferiority of the HA/DBM mixture versus the HA/ $\beta$-TCP mixture, with a two-sided $5 \%$ significance level, a power of $80 \%$, and a noninferiority margin of $15 \%$, a sample size of 36 patients for each group was necessary. Given an anticipated dropout rate of $15 \%$, a total of 85 patients was required.

\section{Statistical analysis}

An independent Student's $t$-test or an analysis of variance test was used for continuous variables and Fisher's exact test was used for proportional variables. SPSS ver. 19.0 (IBM Co., Armonk, NY, USA) was used for all analyses and a two-sided $p$-value $<0.05$ was considered statistically significant.

\section{Results}

\section{Patient characteristics}

Eighty-five eligible patients were randomly assigned to the two groups. Of these, 77 patients ( 38 in group B and 


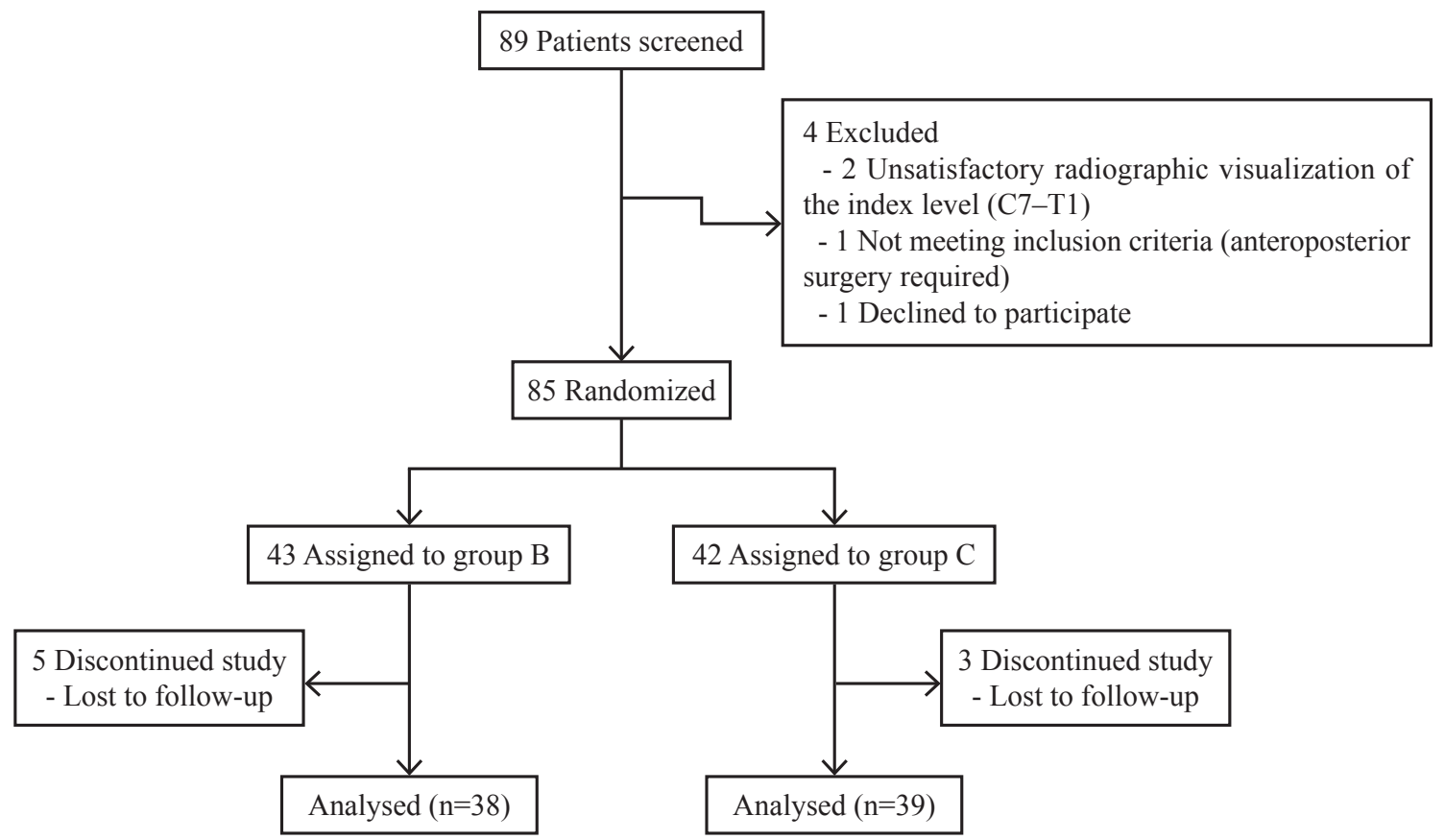

Fig. 4. Flow diagram for the process of enrolling patients.

Table 1. Demographic data

\begin{tabular}{|c|c|c|c|}
\hline Characteristic & Group B (n=38) & Group C (n=39) & $p$-value \\
\hline Age (yr) & $51.9 \pm 11.7$ & $51.3 \pm 12.4$ & 0.71 \\
\hline Gender (male/female) & $25 / 13$ & $26 / 13$ & 0.33 \\
\hline Height (cm) & $165.8 \pm 10.4$ & $162.7 \pm 9.4$ & 0.98 \\
\hline Weight (kg) & $68.8 \pm 13.4$ & $65.3 \pm 11.4$ & 0.33 \\
\hline Body mass index $\left(\mathrm{kg} / \mathrm{m}^{2}\right)$ & $24.9 \pm 2.9$ & $24.6 \pm 3.4$ & 0.40 \\
\hline Smoking status & & & 0.29 \\
\hline Smoker & $13(34)$ & $11(28)$ & \\
\hline Non-smoker & $25(66)$ & $28(72)$ & \\
\hline Clinical manifestation & & & 0.75 \\
\hline Radiculopathy & $25(66)$ & $24(62)$ & \\
\hline Myelopathy & $9(24)$ & $8(20)$ & \\
\hline Myeloradiculopathy & $4(10)$ & $7(18)$ & \\
\hline Fusion level & & & 0.97 \\
\hline $\mathrm{C} 3 / 4$ & $0(0)$ & $1(3)$ & \\
\hline $\mathrm{C} 4 / 5$ & $5(13)$ & $5(13)$ & \\
\hline $\mathrm{C} 5 / 6$ & $18(48)$ & $18(46)$ & \\
\hline $\mathrm{C} 6 / 7$ & $13(34)$ & $15(38)$ & \\
\hline $\mathrm{C} 7 / \mathrm{T} 1$ & $2(5)$ & $0(0)$ & \\
\hline
\end{tabular}

Values are presented as mean \pm standard deviation or number (\%).

39 in group C) fulfilled the criteria for study evaluation (Fig. 4). There were no statistically significant differences between the groups in demographic characteristics, in- cluding age, gender, smoking status, height, weight, BMI, proportion of myelopathy versus radiculopathy, and fusion levels (Table 1). 
Table 2. Fusion rate on dynamic radiographs and computed tomography scan

\begin{tabular}{|c|c|c|c|c|}
\hline Postoperative time (mo) & Diagnostic modality & Group B (n=38) & Group C (n=39) & $p$-value \\
\hline \multirow[t]{2}{*}{3} & Dynamic radiographs & $69\left(25 / 36^{\mathrm{a}}\right)$ & $74\left(29 / 38^{\mathrm{a})}\right)$ & 0.60 \\
\hline & Computed tomography & - & - & - \\
\hline \multirow[t]{2}{*}{6} & Dynamic radiographs & $76(29 / 38)$ & $74(29 / 39)$ & 1.00 \\
\hline & Computed tomography & $74(28 / 38)$ & $67(26 / 39)$ & 0.62 \\
\hline \multirow[t]{2}{*}{12} & Dynamic radiographs & $87(33 / 38)$ & $87(34 / 39)$ & 1.00 \\
\hline & Computed tomography & $87(33 / 38)$ & $72(28 / 39)$ & 0.16 \\
\hline
\end{tabular}

Values are presented as \% (number/total number).

${ }^{a}$ Two patients in group $B$ and one patient in group $C$ were excluded because of insufficient image quality.

Table 3. Pain intensity

\begin{tabular}{llll}
\hline Clinical parameter & Group B (n=38) & Group C (n=39) & $p$-value \\
VAS for posterior neck pain & & & \\
\hline Preoperative & $3.2 \pm 2.9$ & $3.5 \pm 3.2$ & 0.93 \\
\hline 1 mo postoperative & $2.1 \pm 1.8$ & $2.1 \pm 1.9$ & 0.81 \\
\hline 3 mo postoperative & $1.5 \pm 1.8$ & $1.9 \pm 2.2$ & 0.27 \\
\hline 6 mo postoperative & $2.0 \pm 2.4$ & $2.3 \pm 2.4$ & 0.67 \\
\hline 12 mo postoperative & $1.6 \pm 2.1$ & $1.7 \pm 2.2$ & 0.82 \\
\hline VAS for radiating pain in the upper extremity & & & \\
\hline Preoperative & $5.8 \pm 2.2$ & $5.9 \pm 2.7$ & 0.85 \\
\hline 1 mo postoperative & $2.8 \pm 2.3$ & $2.7 \pm 2.3$ & 0.82 \\
\hline 3 mo postoperative & $2.8 \pm 2.7$ & $2.0 \pm 2.0$ & 0.33 \\
\hline 6 mo postoperative & $2.9 \pm 2.5$ & $2.4 \pm 2.3$ & 0.31 \\
\hline 12 mo postoperative & $1.6 \pm 2.1$ & $2.3 \pm 1.9$ & 0.27 \\
\hline
\end{tabular}

Values in data cells represent mean \pm standard deviation.

VAS, visual analogue scale.

\section{Primary endpoint (fusion rate)}

On evaluating the dynamic radiographs three months after surgery, two patients in group B and one patient in group $C$ were excluded because of insufficient image quality; fusion was achieved in $25 / 36$ patients (69\%) in group B and in 29/38 patients (74\%) in group C (Table 2). The difference was not statistically significant $(p=0.60)$. Six months postoperatively, $29 / 38$ patients (76\%) in group B and 29/39 patients (74\%) in group C had achieved fusion, with no statistically significant difference $(p=1.00)$. At 12 months after surgery, bone fusion was achieved in $33 / 38$ patients (87\%) in group B and 34/39 patients (87\%) in group $C$, with no significant difference between the groups $(p=1.00)$.

CT scans taken six months postoperatively showed fusion in 28/38 patients (74\%) in group B and 26/39 patients (67\%) in group C (Table 2). The difference was not statistically significant $(p=0.62)$. At 12 months after surgery, 33/38 patients (87\%) in group B and 28/39 patients (72\%) in group C had achieved fusion on CT scans, with no statistically significant difference $(p=0.16)$.

\section{Secondary endpoints}

The VAS scores for posterior neck pain indicated that pain levels at 12 months after surgery were significantly lower than those at preoperative levels in both groups: the mean scores decreased from $3.2 \pm 2.9$ preoperatively to $1.6 \pm 2.112$ months postoperatively in group $B$, and from $3.5 \pm 3.2$ to $1.7 \pm 2.2$ in group $C(p=0.0035$ and 0.0021 , respectively) (Table 3 ). The scoring of radiating pain in- 
Table 4. Neck disability index score

\begin{tabular}{lccc} 
Clinical parameter & Group B $(\mathrm{n}=38)$ & Group C (n=39) & $p$-value \\
\hline Preoperative & $39 \pm 16$ & $38 \pm 16$ & 0.83 \\
\hline 1 mo postoperative & $26 \pm 16$ & $22 \pm 14$ & 0.31 \\
\hline mo postoperative & $21 \pm 16$ & $21 \pm 13$ & 0.85 \\
6 mo postoperative & $21 \pm 17$ & $20 \pm 13$ & 0.87 \\
12 mo postoperative & $17 \pm 15$ & $18 \pm 15$ & 0.62 \\
\hline
\end{tabular}

Values in data cells represent mean \pm standard deviation.

Table 5. Laboratory tests of inflammatory profiles

\begin{tabular}{lccc} 
Characteristic & Group B $(\mathrm{n}=38)$ & Group C $(\mathrm{n}=39)$ & $p$-value \\
$\begin{array}{l}\text { Inflammatory profiles at one month postoperatively } \\
\text { Erythrocyte sedimentation rate }(\mathrm{mm} / \mathrm{hr})\end{array}$ & $17 \pm 15$ & $15 \pm 13$ & \\
\hline C-reactive protein $(\mathrm{mg} / \mathrm{dL})$ & $0.18 \pm 0.47$ & $0.25 \pm 0.88$ & 0.45 \\
\hline White blood cell count $\left(10^{3} / \mathrm{\mu L}\right)$ & $6570 \pm 1840$ & $6240 \pm 1480$ & 0.36 \\
\hline
\end{tabular}

tensity in the upper extremity at 12 months after surgery also improved significantly in both groups: the mean scores decreased from $5.8 \pm 2.2$ preoperatively to $1.6 \pm 2.1$ one year postoperatively in group $\mathrm{B}$, and from $5.9 \pm 2.7$ to $2.3 \pm 1.9$ in group $C(p<0.0001$ and $<0.0001$, respectively). There was no statistically significant difference between the two groups in terms of posterior neck pain and radiating pain, both preoperatively and at all time points during the follow-up period (Table 3 ).

The mean NDI scores were similar in both groups at baseline, at $39 \pm 16$ in group B and $38 \pm 16$ in group C, with no significant difference ( $p=0.83$ ) (Table 4$)$. Patients in both groups showed a marked improvement in NDI scores from baseline at one year's follow-up, which was $17 \pm 15$ in group B and $18 \pm 15$ in group C. There was no statistically significant difference between the two groups in NDI score both preoperatively and at all time points during follow-up (Table 4).

One month after surgery, the laboratory examination of inflammatory profiles revealed that in group B, ESR was $17 \pm 15 \mathrm{~mm} / \mathrm{hr}$, CRP was $0.18 \pm 0.47 \mathrm{mg} / \mathrm{dL}$, and $\mathrm{WBC}$ was $6570 \pm 1840 \times 10^{3} / \mu \mathrm{L}$; and in group C, ESR was $15 \pm 13 \mathrm{~mm} /$ $\mathrm{hr}, \mathrm{CRP} 0.25 \pm 0.88 \mathrm{mg} / \mathrm{dL}$ and WBC $6240 \pm 1480 \times 10^{3} / \mu \mathrm{L}$ (Table 5). There was no statistically significant differences between the groups ( $p=0.45,0.36$, and 0.23 , respectively). There were no patients with postoperative infections in either group.

\section{Discussion}

Fusion status has been widely accepted as a critical prognostic factor in ACDF $[2,8,11,14,22-24]$. Therefore, spine surgeons have endeavored to find better graft materials or substitutes to achieve solid fusion. In order to avoid the drawbacks of autografts and allografts, several synthetic cages, including metal, carbon fiber, and PEEK cages, have been developed to maximize outcomes while minimizing complications. The ideal synthetic cage for ACDF needs to provide immediate stability, achieve a high fusion rate, and reduce the complications associated with autografts and allografts. The PEEK cage has several strengths compared to other synthetic cages. Its elastic modulus is similar to that of human bone, leading to lower cage subsidence and better load distribution between the bone and the cage [7-9,25]. Owing to its radiolucency, postoperative fusion status can be easily evaluated with radiographs, and implant artifact on postoperative CT or MRI scans is diminished. In addition, PEEK cages can induce the attachment of osteogenic cells and fibroblast proliferation $[8,25]$. However, the PEEK cage does not have the necessary properties to induce bony regeneration, such as osteoconduction and osteoinduction. To overcome this limitation, filling materials such as HA, $\beta$-TCP, and DBM have been used inside the PEEK cage. Synthetic materials such as HA and $\beta$-TCP have different properties from graft substitutes such as 
DBM: the former is osteoconductive, whereas the latter is osteoinductive $[1,4,5,7,10-14,18,26]$. A material fully equipped with all of these properties could provide better fusion outcomes; therefore, mixing materials with different properties might enhance fusion rates. However, few clinical trials have been performed in this regard.

In a review of the literature we could not find any reports on the use of a mixture of HA and DBM inside a PEEK cage. One animal study and one clinical article each reported on the use of a mixture of allograft bone chips and DBM to enhance bony union in ACDF, with favorable outcomes $[3,27]$. However, drawbacks of the mixture included its high cost and allograft-related problems. We therefore evaluated a mixture of HA and DBM as a PEEK cage filler in ACDF, and this is the first study to investigate the outcomes of such a process. This was a prospective randomized study comparing these outcomes with those of a PEEK cage filled with a mixture of HA and $\beta$-TCP. This study showed that the HA/ DBM mixture provides noninferior outcomes in terms of fusion rates and clinical outcomes compared with the HA/ $\beta$-TCP mixture. Fusion rates were not significantly different between the two cages. For example, the fusion rate at 12 months postoperatively was $87 \%$ in each group on dynamic radiographs, and $87 \%$ in group $\mathrm{B}$ versus $72 \%$ in group C on CT scans, with no statistically significant difference between the groups ( $p=1.00$ and 0.16 , respectively). Clinically, there were no statistically significant differences between the two groups with regard to improvements in pain intensity, NDI score, postoperative inflammatory profiles, and infection rates. Therefore, we concluded that the mixture of HA/DBM in a PEEK cage was not inferior to the mixture of HA/ $\beta$-TCP for ACDF.

In the current study the fusion rate was slightly lower than in previous reports. This is thought to be caused by our very strict criteria for bony union. The measurement with dynamic radiographs was carried out strictly according to our criteria for the definite evaluation of fusion status. In addition, even a little or vague discontinuity on CT scans was judged to be a nonunion.

This study has a limitation, in that its noninferiority design means that the sample size was relatively small. A superiority study design with a larger sample size might better compare the clinical and radiological outcomes of the two mixtures.

\section{Conclusions}

In this study, the mixture of HA and DBM was evaluated as a fusion material in a PEEK cage and compared with a mixture of HA and $\beta$-TCP in a prospective randomized design. There was no statistically significant difference between the fusion materials in the union rate, which was the primary endpoint of this noninferiority trial. In addition, there were no significant differences in secondary endpoints, such as pain at the posterior neck and upper extremity, NDI score, laboratory tests of inflammatory profiles, and infection rate. Based on the results, the authors concluded that the HA/DBM mixture as a fusion material in a PEEK cage would provide noninferior outcomes compared to the HA/ $\beta$-TCP mixture, and could be a useful alternative.

\section{Acknowledgments}

This work was supported by a grant of the Small and Medium Business Administration, Republic of Korea (MD320359).

We thank and acknowledge Ms. Junga Park, Song Yi Kim, Sun Yong Lee, and Yoon Ju Kwon, spine research coordinators at our institution, and Ms. Young Hee Ahn, a physician assistant, for their contributions to the project.

\section{References}

1. Fischer CR, Cassilly R, Cantor W, Edusei E, Hammouri Q, Errico T. A systematic review of comparative studies on bone graft alternatives for common spine fusion procedures. Eur Spine J 2013;22:142335.

2. Chen F, He W, Mahaney K, et al. Alternative grafts in anterior cervical fusion. Clin Neurol Neurosurg 2013; 115:2049-55.

3. Urrutia J, Thumm N, Apablaza D, Pizarro F, Zylberberg A, Quezada F. Autograft versus allograft with or without demineralized bone matrix in posterolateral lumbar fusion in rabbits. Laboratory investigation. J Neurosurg Spine 2008;9:84-9.

4. Giannoudis PV, Dinopoulos H, Tsiridis E. Bone substitutes: an update. Injury 2005;36 Suppl 3:S20-7.

5. Vaccaro AR, Chiba K, Heller JG, et al. Bone grafting alternatives in spinal surgery. Spine J 2002;2:206-15. 
6. Zimmermann G, Moghaddam A. Allograft bone matrix versus synthetic bone graft substitutes. Injury 2011;42 Suppl 2:S16-21.

7. Chau AM, Xu LL, Wong JH, Mobbs RJ. Current status of bone graft options for anterior interbody fusion of the cervical and lumbar spine. Neurosurg Rev 2014;37:23-37.

8. Cabraja M, Oezdemir S, Koeppen D, Kroppenstedt S. Anterior cervical discectomy and fusion: comparison of titanium and polyetheretherketone cages. BMC Musculoskelet Disord 2012;13:172.

9. Chen Y, Wang X, Lu X, et al. Comparison of titanium and polyetheretherketone (PEEK) cages in the surgical treatment of multilevel cervical spondylotic myelopathy: a prospective, randomized, control study with over 7-year follow-up. Eur Spine J 2013;22:153946.

10. Cheng L, Ye F, Yang R, et al. Osteoinduction of hydroxyapatite/beta-tricalcium phosphate bioceramics in mice with a fractured fibula. Acta Biomater 2010;6: 1569-74.

11. Cosar M, Ozer AF, Iplikcioglu AC, et al. The results of beta-tricalcium phosphate coated hydroxyapatite (beta-TCP/HA) grafts for interbody fusion after anterior cervical discectomy. J Spinal Disord Tech 2008;21:436-41.

12. Lee KJ, Roper JG, Wang JC. Demineralized bone matrix and spinal arthrodesis. Spine J 2005;5:217S-223S.

13. Yoshii T, Yuasa M, Sotome S, et al. Porous/dense composite hydroxyapatite for anterior cervical discectomy and fusion. Spine (Phila Pa 1976) 2013;38:83340.

14. Sugawara T, Itoh Y, Hirano Y, Higashiyama N, Mizoi K. $\beta$-Tricalcium phosphate promotes bony fusion after anterior cervical discectomy and fusion using titanium cages. Spine (Phila Pa 1976) 2011;36:E150914.

15. Aghdasi B, Montgomery SR, Daubs MD, Wang JC. A review of demineralized bone matrices for spinal fusion: the evidence for efficacy. Surgeon 2013;11:3948.

16. Pietrzak WS, Perns SV, Keyes J, Woodell-May J, McDonald NM. Demineralized bone matrix graft: a scientific and clinical case study assessment. J Foot Ankle Surg 2005;44:345-53.

17. Gruskin E, Doll BA, Futrell FW, Schmitz JP, Hollinger JO. Demineralized bone matrix in bone repair: history and use. Adv Drug Deliv Rev 2012;64:106377.

18. Veillette CJ, McKee MD. Growth factors--BMPs, DBMs, and buffy coat products: are there any proven differences amongst them? Injury 2007;38 Suppl 1:S38-48.

19. Drosos GI, Kazakos KI, Kouzoumpasis P, Verettas DA. Safety and efficacy of commercially available demineralised bone matrix preparations: a critical review of clinical studies. Injury 2007;38 Suppl 4:S1321.

20. Piaggio G, Elbourne DR, Pocock SJ, Evans SJ, Altman DG; CONSORT Group. Reporting of noninferiority and equivalence randomized trials: extension of the CONSORT 2010 statement. JAMA 2012;308:2594604.

21. Chou YC, Chen DC, Hsieh WA, et al. Efficacy of anterior cervical fusion: comparison of titanium cages, polyetheretherketone (PEEK) cages and autogenous bone grafts. J Clin Neurosci 2008;15:1240-5.

22. Kong CG, Kim YY, Ahn CY, Park JB. Diagnostic usefulness of white blood cell and absolute neutrophil count for postoperative infection after anterior cervical discectomy and fusion using allograft and demineralized bone matrix. Asian Spine J 2013;7:173-7.

23. Dagli M, Er U, Simsek S, Bavbek M. Late results of anterior cervical discectomy and fusion with interbody cages. Asian Spine J 2013;7:34-8.

24. Ahn JS, Lee JK, Kim JH. Comparative study of clinical outcomes of anterior cervical discectomy and fusion using autobone graft or cage with bone substitute. Asian Spine J 2011;5:169-75.

25. Chang MY, Chen MH, Chang CJ, Huang JS. Preliminary clinical experience with polyetheretherketone cages filled with synthetic crystallic semihydrate form of calcium sulfate for anterior cervical discectomy and fusion. Formos J Surg 2013;46:109-15.

26. Lee JH, Ryu MY, Baek HR, et al. Effects of porous beta-tricalcium phosphate-based ceramics used as an E. coli-derived rhBMP-2 carrier for bone regeneration. J Mater Sci Mater Med 2013;24:2117-27.

27. An HS, Simpson JM, Glover JM, Stephany J. Comparison between allograft plus demineralized bone matrix versus autograft in anterior cervical fusion. A prospective multicenter study. Spine (Phila Pa 1976) 1995;20:2211-6. 